\title{
PROPAGATION OF UNEQUAL OTDM DATA CHANNELS IN 2R REGENERATED SYSTEM
}

Zhijian Huang, Ashley Gray, Igor Khrushchev, Ian Bennion

Photonics Research Group, Electronic Engineering,

Aston University, Birmingham, B4 7ET, United Kingdom

E-mail: z.huang@aston.ac.uk

Abstract: Switching of an optical signal comprising individual OTDM channels of unequal amplitudes in a nonlinear optical loop mirror is investigated. The propagation dynamics of unequal-channel data streams in a switch-guided, dispersion-managed link are also studied

\section{INTRODUCTION}

Optical time division multiplexing (OTDM) is currently the only way to form optical data streams at ultrahigh speeds of $80 \mathrm{~Gb} / \mathrm{s}$ and above. Due to the finite accuracy of channel equalisation, some variations of amplitude between the channels is inevitable in OTDM transmitters. Other fundamental effects, such as interferometric noise induced by finite pulse extinction ratio and phase noise from the laser source [1] also contribute to these variations. Moreover, it is sometimes advantageous, from the system point of view, to introduce the channel inequality deliberately either to reduce the pulse-to-pulse interaction[2] or to simplify the task of clock recovery [3].

All $2 \mathrm{R}$ regenerators, including nonlinear optical fibre loop mirrors, NOLMs [46], possess nonlinear transmission response characteristics. Therefore, OTDM channels of different amplitude will experience different transformations when switched in a $2 \mathrm{R}$ device. This is an important factor in a $2 \mathrm{R}$-supported data transmission link. In this paper, we investigate how the channel inequality affects the nonlinear signal switching in a $2 \mathrm{R}$ regenerator and the data transmission in a nonlinear switch-guided system. 


\section{EXPERIMENTAL SETUP}

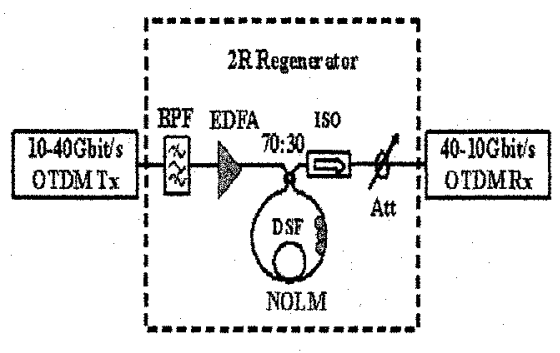

(a)

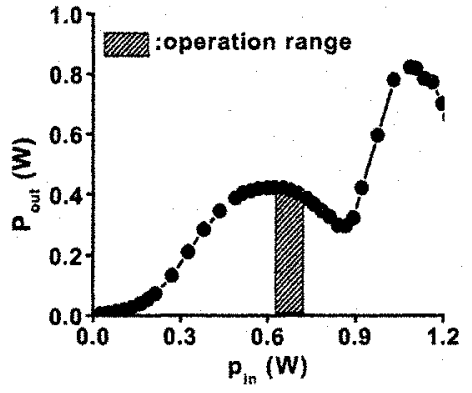

(b)

Fig. 1 a) Experimental setup b) Switching curve of NOLM.

The NOLM shown in Fig. 1(a) comprised a 70:30 fibre coupler and $2.3 \mathrm{~km}$ of dispersion shifted fibre with an anomalous dispersion of $+2.8 \mathrm{ps} /(\mathrm{nm} \cdot \mathrm{km})$ at the wavelength of $1550 \mathrm{~nm}$. A mode-locked fibre laser generated Gaussian-shaped 3.5ps pulses with an extinction ratio higher than $33 \mathrm{~dB}$. The pulses were modulated at $10 \mathrm{Gbit} / \mathrm{s}$ by a pseudo-random bit stream (PRBS) of length $2^{31}-1$ and subsequently optically multiplexed to form a single-polarization, 40Gbit/s OTDM data stream by using a two-stage fibre delay line. It was possible to vary the amplitudes of the four OTDM channels in the $40 \mathrm{Gbit} / \mathrm{s}$ signal individually by adjusting the polarization controllers inside the delay line. At the receiver, the $40 \mathrm{Gbit} / \mathrm{s}$ signal was optically de-multiplexed to $10 \mathrm{Gbit} / \mathrm{s}$ using an electro-absorption modulator.

\section{RESULTS AND DISCUSSION}

Figure 1(b) shows the measured transmission of the NOLM as a function of average power of the input $40 \mathrm{~Gb} / \mathrm{s}$ signal. The shaded area indicates the input optical power range that usually provides the best switching and noise suppression performance $[4,5]$.

The switching behaviour of the OTDM signal was investigated by using histogram analysis of the eye diagrams taken on a $50 \mathrm{GHz}$ digital sampling oscilloscope equipped with a $32 \mathrm{GHz}$ photo-detector. The signals were measured before and after the NOLM. We used the relative standard deviation of amplitude, $\Delta \mathrm{V}$, as a measure of inter-channel non-uniformity. The mean voltage value $V_{i}$ $(i=1,2,3,4)$ of marks in each individual eye determined the amplitude of a corresponding channel. We defined the inter-channel amplitude difference as $\Delta V=\sigma / \mu$, where $\sigma$ is the standard deviation of $V$ measured channel-to-channel and $\mu$ is the averaged value of $V$ over the four channels. 

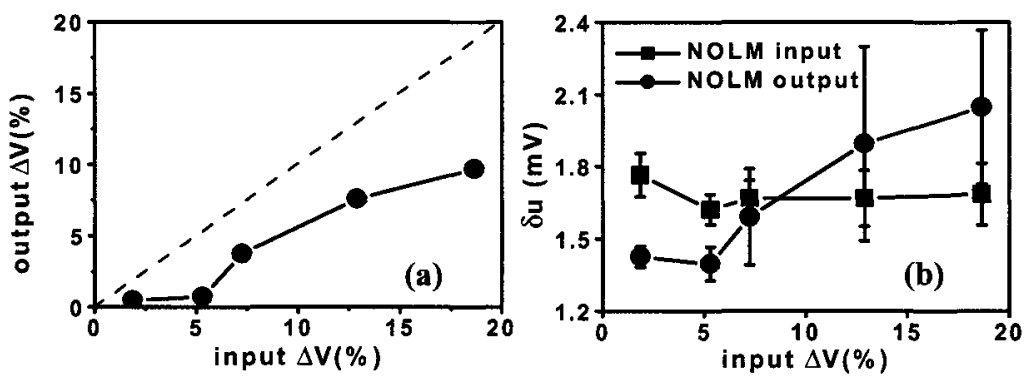

Fig. 2 a) Inter-channel amplitude variation, $\Delta V$, before and after the NOLM. b) Random amplitude fluctuations $\delta \mathrm{u}$ before (squares) and after (circles) the NOLM, depending on the input $\Delta \mathrm{V}$.

The input $\Delta \mathrm{V}$ was varied by adjustment of the OTDM multiplexer. The average power at the NOLM input was optimised in order to minimize the $\Delta \mathrm{V}$ of the signal at the NOLM output. The average power arriving at the photo-detector was maintained constant. Fig. 2(a) shows the output $\Delta \mathrm{V}$ as a function of that at the NOLM input. One can see that the inter-channel amplitude variation is always reduced by the NOLM, but the effect becomes weaker as the input signal becomes less uniform.

We also studied the dynamics of another important parameter, the random fluctuation of amplitude. This was characterised by $\delta u=\left(\Sigma \rho_{1}\right) / 4$, where $\rho_{\mathrm{i}}$ was the standard deviation of amplitude within each channel.

Fig. 2(b) shows $\delta u$ as a function of the input amplitude difference, $\Delta V$, measured before and after the NOLM. The height of the error bar indicates the difference between the minimum and maximum $\rho_{i}$ measured in the most stable and in the noisiest of the four channels, respectively. One can see that the effect of the input channel non-uniformity on the amplitude noise suppression is significant. When the input amplitudes are relatively uniform ( $\Delta \mathrm{V}$ less than $5 \%$ in the experiment), the NOLM efficiently suppresses the amplitude fluctuations in all channels. However, when the input channels become considerably unequal $(\Delta \mathrm{V}$ larger than 10\%), the efficiency of the amplitude noise suppression strongly varies among the channels. As a result, the overall quality of the switched signal deteriorates. In fact, a signal comprising very non-uniform OTDM channels actually experiences an amplitude noise increase as a result of switching in the NOLM.

Overall, the inter-channel amplitude difference is usually reduced by the NOLM, whilst amplitude fluctuations of individual channels may be either suppressed or increased depending on the non-uniformity of the input signal. 


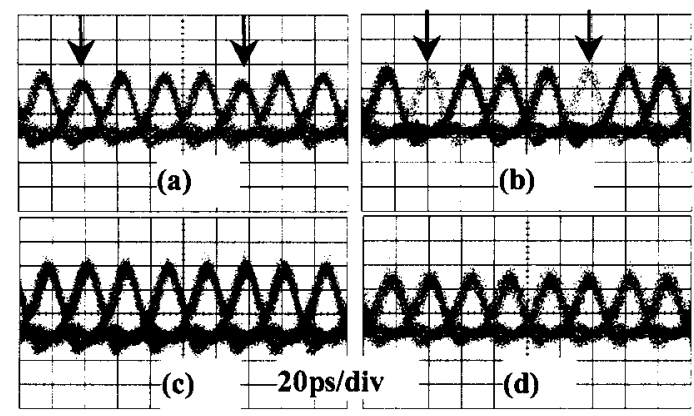

Fig.3. Eye diagrams. a) Initial $\Delta \mathrm{V}=15 \%$, back-to-back. b) $\Delta \mathrm{V}=15 \%$, measured after $2,500 \mathrm{~km}$. c) $\Delta V=5 \%$, back-to-back. d) $\Delta V=5 \%$, after $2,500 \mathrm{~km}$.

Non-uniformity of the input signal is likely to affect the data transmission in a system employing NOLMs as in-line $2 \mathrm{R}$ regenerators. If the format of the input signal is such that the amplitude fluctuations in some channels increase in the first NOLM, these channels will subsequently deteriorate during propagation through subsequent $2 \mathrm{R}$ elements, probably resulting in unstable propagation of corresponding channels.

We studied the effect of channel non-uniformity on the data transmission by monitoring the eye diagrams and $\mathrm{Q}$-factor of a digital signal propagating in a recirculating loop with the in-line NOLM acting as a $2 \mathrm{R}$ element [6]. The $2 \mathrm{R}$ regeneration was performed after every $200 \mathrm{~km}$ of fibre. The Q-factor was calculated by using decision threshold measurements [7]. Figure 3 shows the eye diagrams measured back-to-back and after propagating over $2500 \mathrm{~km}$ of SMF. The initial amplitude difference, $\Delta \mathrm{V}$, was set at a level of either $15 \%$ (Fig.3(a,b)) or $5 \%$ (Fig.3(c,d)) for this experiment.

With an initial $\Delta V$ of $15 \%$ (Fig.3a), the signal considerably deteriorated during transmission, showing drop-out of the selected channel after propagating over $2,500 \mathrm{~km}$ (Fig. $3 \mathrm{~b}$ ). The overall bit-error-rate of the $40 \mathrm{~Gb} / \mathrm{s}$ data stream increased dramatically during the first several hundred kilometres of transmission.

The higher quality signal with an initial $\Delta \mathrm{V}$ of $5 \%$ (Fig.3c) propagated in a totally different manner. Channel non-uniformity was eliminated after multiple transmissions through the $2 \mathrm{R}$ element, resulting in a virtually perfectly uniform OTDM data stream observed after $2,500 \mathrm{~km}$ of propagation (Fig.3d). The estimated Q-factor was as high as $19 \mathrm{~dB}$ after $2500 \mathrm{~km}$ of SMF (Fig.4) and the error-free propagation (Q 15.6dB) distance was estimated to be in excess of 5800 $\mathrm{km}$. 


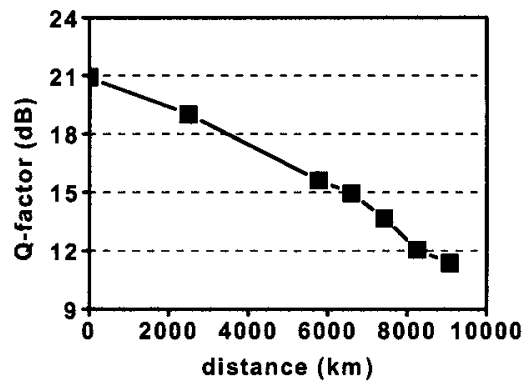

Fig.4. Q-factor vs. transmission distance.

\section{CONCLUSION}

We have shown that channel-to-channel amplitude differences in OTDM data streams have a strong impact on the switching behaviour of individual channels in a 2R-regenerator. Depending on the inter-channel amplitude difference, the optical pulses in different channels experience either suppression of the amplitude noise, or a noise increase. Appropriate control of the channel uniformity in the OTDM transmitters is necessary in order to support stable long haul transmission in $2 \mathrm{R}$ regenerated systems.

\section{REFERENCES}

[1] I. Y. Khrushchev, et al, "OTDM applications of dispersion-imbalanced fibre loop mirror”, Electron. Lett., , vol. 35, pp. 1183-1185, 1999

[2] G. P. Agrawal, Nonlinear Fiber Optics, Academic Press, 1989), 130-133

[3] A. H. Gnauck, et al " $1-\mathrm{Tb} / \mathrm{s}(6 \times 170.6 \mathrm{Gbit} / \mathrm{s})$ transmission over $2000-\mathrm{km}$ NZDF using OTDM and RZ-DPSK format", IEEE Photon. Technol. Lett., vol. 15, pp. 1618-1620, Nov., 2003

[4] N. J. Smith et al, "Picosecond soliton transmission using concatenated nonlinear optical loop-mirror intensity filters", J. Opt. Soc. Am. B, vol. 12, pp. 1117-1125, 1995

[5] S. Boscolo, et al "Study of the operating regime for all-optical passive $2 \mathrm{R}$ regeneration of dispersion-managed RZ data at $40 \mathrm{~Gb} / \mathrm{s}$ using in-line NOLMs", IEEE Photon. Technol. Lett. , vol.14, pp. 30-33, Jan., 2002

[6] Z. Huang, et al " $40 \mathrm{~Gb} / \mathrm{s}$ transmission over $4000 \mathrm{~km}$ of standard fibre using in-line nonlinear optical loop mirrors", in Proceedings of the 29th European Conference on Optical Communication (ECOC/IOOC 2003), Rimini, Italy, paper Mo4.6.3, 21-25 September 2003

[7] N. S. Bergano, F. W. Kerfoot, and C. R. Davidson, "Margin measurements in optical amplifier systems," IEEE Photonics Technol. Lett, vol. 5 pp. 304-306, Mar. 1993 